\title{
Preperimetric glaucoma diagnosis by confocal scanning laser tomography of the optic disc
}

\author{
Christian Y Mardin, Folkert K Horn, Jost B Jonas, Wido M Budde
}

\begin{abstract}
Aim-To evaluate the ability of confocal scanning laser tomography of the optic nerve head to detect glaucomatous optic nerve damage in ocular hypertensive eyes without visual field defects.

Methods-The study included 50 normal subjects, 61 glaucoma patients with glaucomatous changes in the optic disc and visual field, and 102 "preperimetric" patients with increased intraocular pressure, normal visual fields, and glaucomatous appearance of the optic disc as evaluated on colour stereo optic disc photographs. For all individuals, confocal scanning laser tomographs of the optic nerve head were taken using the Heidelberg retina tomograph (HRT; software
\end{abstract} 2.01).

Results-Almost all investigated HRT variables varied significantly $(p<0.05)$ between the normal eyes and preperimetric glaucoma eyes with pronounced overlap between the two study groups. Corresponding to the overlap, sensitivity and specificity values were relatively low when HRT variables were taken to differentiate between normal and preperimetric glaucoma eyes. At a given specificity of $95 \%$ highest sensitivities were found for the variables "rim area in the superior disc sector" (24.8\%), "nerve fibre layer thickness in the inferior disc sector" $(26.5 \%)$, and "rim volume in the superior disc sector" $(25.5 \%)$. A multivariate approach increased sensitivity to $42.2 \%$ at a given specificity of $95 \%$. For the glaucoma group highest sensitivity values were reached by rim volume in the superior disc sector $(73.8 \%)$ and rim area $(72.1 \%)$; the multivariate approach reached $83.6 \%$.

Conclusions-Owing to pronounced overlapping between the groups, confocal scanning laser tomography of the optic nerve head has relatively low diagnostic power to differentiate between normal eyes and preperimetric glaucoma eyes. One of the reasons may be the biological interindividual variability of quantitative optic disc variables.

(Br F Ophthalmol 1999;83:299-304)

22 September 1998
Glaucoma leads to changes in the intrapapillary and parapapillary region of the optic nerve head such as loss of neuroretinal rim, change of the neuroretinal rim shape, localised and diffuse deepening and widening of the optic cup, disc haemorrhages, chorioretinal atrophy in the parapapillary region, focal and diffuse diminution of the diameter of the retinal arterioles, and localised and diffuse loss of retinal nerve fibre layer. ${ }^{12}$ These morphological alterations can be divided into qualitative criteria such as occurrence of disc haemorrhages, presence of localised retinal nerve fibre layer defects, and occurrence of neuroretinal rim notches, and into quantitative variables such as area and volume of the neuroretinal rim and optic cup, and thickness of the retinal nerve fibre layer at the disc border. ${ }^{3}$ Previous glaucoma studies have shown that in some eyes, the morphological changes can precede visual field defects as measured by conventional computerised static perimetry. ${ }^{3-10}$ In these studies, qualitative criteria have mostly been used to demonstrate the presence of glaucomatous damage of the optic nerve.

The purpose of the present investigation was to evaluate whether quantitative morphological optic disc variables measured by confocal scanning laser tomography are useful for the early detection of glaucomatous optic nerve atrophy in eyes with elevated intraocular pressure, abnormal appearance of the optic disc, and normal visual fields.

\section{Patients and methods}

The study included 102 "preperimetric" patients, 50 normal subjects, and 61 glaucoma patients (Table 1). All "preperimetric" glaucoma patients had an open chamber angle, increased IOP (measurements $>21 \mathrm{~mm} \mathrm{Hg}$ ), an abnormal appearance of the optic nerve head, and normal visual fields examined by conventional computerised perimetry (Octopus programme G1). All patients in the glaucoma group had an open anterior chamber angle, increased intraocular pressure measurements above $21 \mathrm{~mm} \mathrm{Hg}$, an abnormal appearance of the optic disc, and glaucomatous visual field defects. An abnormal appearance of the optic nerve head was defined by the presence of an abnormal shape of the neuroretinal rim including rim notches, localised or diffuse loss of retinal nerve fibre layer, and disc haemorrhages. Mixed together with optic nerve head photographs of more than 500 other normal subjects and patients with non-glaucomatous or glaucomatous optic nerve damage, the optic disc slides were evaluated in a masked fashion by two examiners (WMB and JBJ) without 
Table 2 Morphometric optic disc variables measured by confocal laser scanning tomography (mean (SD)) in three groups: controls, preperimetric open angle glaucoma $(O A G)$, perimetric open angle glaucoma

\begin{tabular}{lllll}
\hline HRT variable & $\begin{array}{l}\text { Disc } \\
\text { region }\end{array}$ & Controls & Preperimetric OAG & Perimetric OAG \\
\hline Rim area $\left(\mathrm{mm}^{2}\right)$ & all & $1.64(0.27)$ & $1.5(0.32)$ & $1.04(0.43)$ \\
& superior & $0.42(0.07)$ & $0.38(0.1)$ & $0.25(0.1)$ \\
Rim volume $\left(\mathrm{mm}^{3}\right)$ & inferior & $0.42(0.09)$ & $0.39(0.09)$ & $0.26(0.12)$ \\
& all & $0.47(0.14)$ & $0.38(0.15)$ & $0.20(0.12)$ \\
& superior & $0.12(0.04)$ & $0.09(0.04)$ & $0.05(0.03)$ \\
Retinal nerve fibre layer thickness $(\mathrm{mm})$ & inferior & $0.12(0.04)$ & $0.10(0.05)$ & $0.05(0.04)$ \\
& all & $0.27(0.06)$ & $0.24(0.07)$ & $0.17(0.07)$ \\
Cup area $\left(\mathrm{mm}^{2}\right)$ & superior & $0.32(0.07)$ & $0.28(0.09)$ & $0.19(0.08)$ \\
& inferior & $0.32(0.07)$ & $0.28(0.09)$ & $1.3(0.5)$ \\
Cup volume $\left(\mathrm{mm}^{3}\right)$ & all & $0.65(0.33)$ & $0.77(0.38)$ & $0.34(0.12)$ \\
& superior & $0.15(0.09)$ & $0.2(0.11)$ & $0.34(0.14)$ \\
Cup shape measure & inferior & $0.16(0.09)$ & $0.19(0.1)$ & $0.13(0.3)$ \\
& all & $0.17(0.12)$ & $0.2(0.2)$ & $0.13(0.09)$ \\
& superior & $0.04(0.03)$ & $0.06(0.06)$ & $-0.06(0.1)$ \\
& inferior & $0.04(0.03)$ & $0.06(0.06)$ & $0.02(0.14)$ \\
& all & $-0.19(0.08)$ & $-0.17(0.08)$ & $0.01(0.14)$ \\
\hline
\end{tabular}

knowledge of the visual field data or IOP measurements. One of the examiners had experience in morphological evaluation of the optic nerve heads of more than 500 patients, the other examiner has been active in this field for more than 10 years. If uncertainty existed in the clinical categorisation of the patients, which was based on one single cross section assessment, the more experienced examiner decided upon the diagnosis. For the evaluation of the retinal nerve fibre layer, black and white wide angle photographs of the retinal nerve fibre layer were additionally available for all patients included in the study.

A glaucomatous visual field defect was defined as a Octopus G1 field with (a) at least three adjacent test points having a deviation of equal or greater than $5 \mathrm{~dB}$ and with one test point with a deviation greater than $10 \mathrm{~dB}$ lower, (b) at least two adjacent test points with a deviation equal to or greater than $10 \mathrm{~dB}$, (c) at least three adjacent test points with a deviation equal to or greater than $5 \mathrm{~dB}$ abutting the nasal horizontal meridian, and (d) a mean visual field defect of more than 2 $\mathrm{dB}$.

The glaucoma group was differentiated into patients with primary open angle glaucoma in

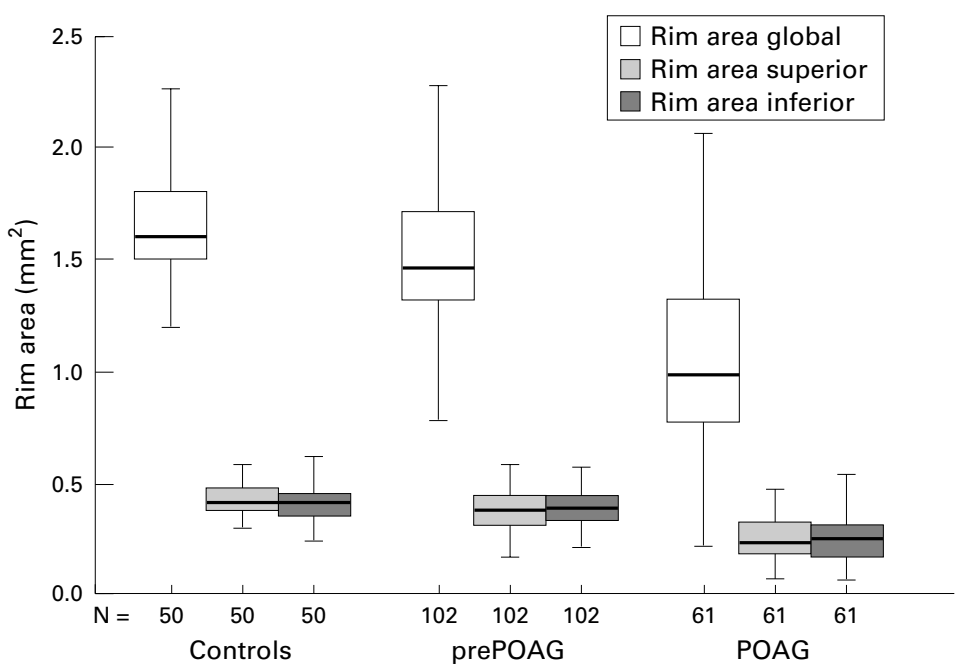

Figure 1 Box plots showing the distribution of neuroretinal rim area in three groups: controls, preperimetric open angle glaucoma (prePOAG), and perimetric open angle glaucoma (POAG). whom no obvious reason for the elevation of IOP had been detected $(n=38)$ and into patients with secondary open angle glaucoma due to pseudoexfoliation of the lens $(n=5)$ or primary pigment dispersion $(n=18)$ (Table 1$)$. To avoid bias by small and large optic discs, disc area was limited to $1.8-2.8 \mathrm{~mm}^{2}$.

Patients with a myopic refractive error exceeding -8 dioptres were excluded owing to different morphology of the optic disc. ${ }^{10}$ If both eyes were examined, only one randomly selected eye per subject was taken into for statistical analysis.

For each eye, three $10^{\circ}$ confocal scanning laser tomographic images were obtained using the Heidelberg retina tomograph (HRT) (Heidelberg Engineering, Heidelberg, Germany, software version 2.01). ${ }^{11}{ }^{12}$ The mean topography of the three images was analysed by the HRT software version 2.01, as proposed by Burk and co-workers. ${ }^{12}$ Mean deviation of the images used in this study was 24.1 (SD 7.8) $\mu \mathrm{m}$. The border of the disc was outlined manually with the help of the optic disc photographs projected simultaneously. According to software 2.01, reference plane for the delineation of the optic cup from the neuroretinal rim was the level $50 \mu \mathrm{m}$ beneath the contour line in the temporal segment, 4-10 degrees below the horizontal axis of the disc. We determined the HRT variables disc area, volume, and area of the neuroretinal rim (volume/area above reference plane) and volume and area of the optic cup (volume/area below reference plane), cup shape measure (third central moment of the frequency distribution of depth values, relative to the curved surface of all parts in the contour line the steeper the cup walls, the more positive the value), and thickness of the retinal nerve fibre layer (measured along the contour line). The HRT variables were measured for the optic disc as a whole, in a right angled superior sector and a right angled inferior sector. The middle lines of both sectors were tilted $13^{\circ}$ temporal to the vertical optic disc axis. For all eyes, $15^{\circ}$ colour stereo optic disc transparencies were additionally taken. ${ }^{13}$ HRT examination and optic disc photography were performed on the same day. 


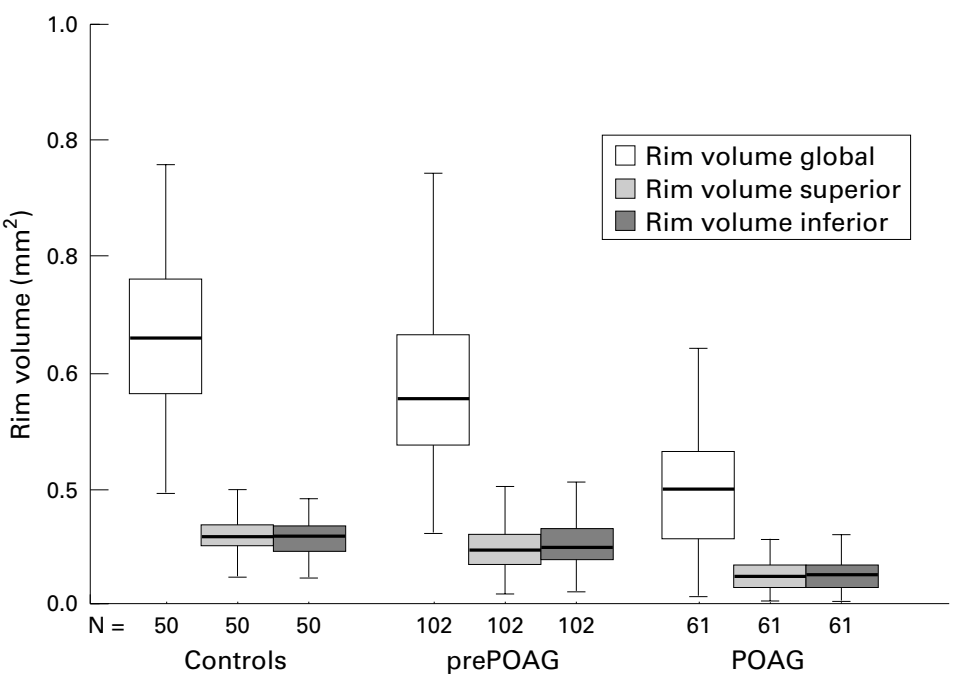

Figure 2 Box plots showing the distribution of neuroretinal rim volume above the reference level in three groups: controls, preperimetric open angle glaucoma (prePOAG), and perimetric open angle glaucoma (POAG).

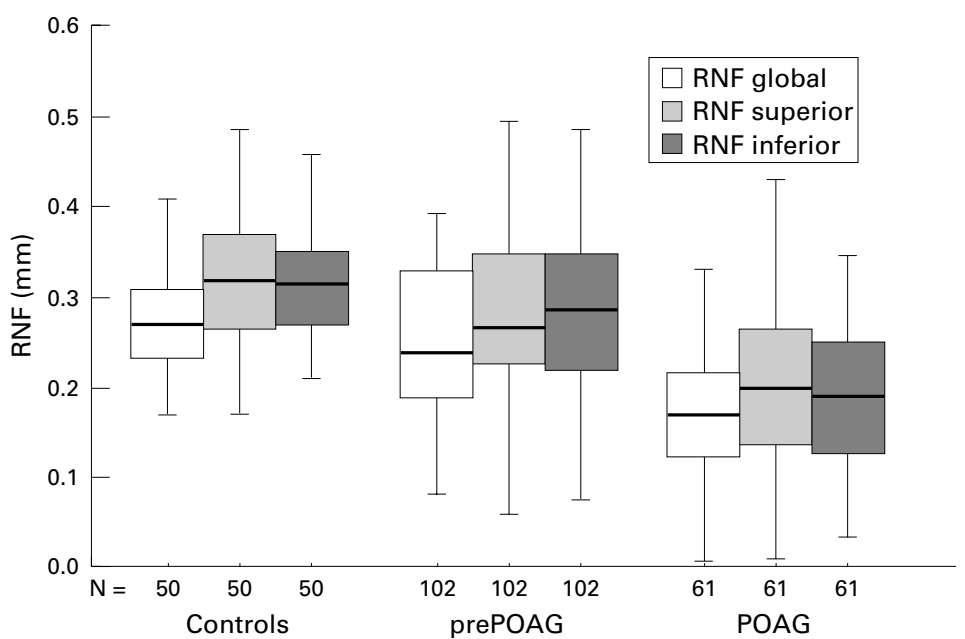

Figure 3 Box plots showing the distribution of retinal nerve fibre (RNF) layer thickness in three groups: controls, preperimetric open angle glaucoma (prePOAG), and perimetric open angle glaucoma (POAG).

\section{Results}

All tested HRT variables were significantly ( $p<0.05$; ranked Mann-Whitney U test) different between the normal group and the "preperimetric" glaucoma group, with the exception of the cup shape measure, measured globally and separately in the inferior disc sector, rim area in the inferior sector, cup area and volume in the inferior sector and global cup (Table 2). Parallel to marked interindividual variability in each group, there was pronounced overlap in all measured optic disc variables between the two study groups (Table 2) (Figs 1-3).

To differentiate between the normal eyes and the preperimetric glaucoma eyes, neuroretinal rim area in the superior sector $(24.8 \%)$, thickness of the retinal nerve fibre layer in the inferior sector $(26.5 \%)$, and rim volume above the reference level in the inferior sector $(25.5 \%)$ showed highest sensitivity values at $95 \%$ specificity (Table 3 ).

Comparing the normal eyes with the glaucoma eyes with perimetric defects, all HRT variables were significantly $(p<0.001)$ different, again with marked overlap between the study groups (Table 2) (Figs 1-3). Neuroretinal rim area in the superior sector $(72.1 \%)$, rim volume above the reference plane global $(67.2 \%)$, and in the superior sector $(73.8 \%)$ showed highest sensitivity values at $95 \%$ specificity. The cup shape measure in the inferior sector $(26.2 \%)$ showed lowest sensitivity (Table 3).

For further separation between the normal eyes and the glaucoma eyes with perimetric defects, a discriminant function was derived for multivariate presentation. The function coefficients of the present study are given in the formula of the value (D) of the discriminant function: $\mathrm{D}=-2.77+0.3 \mathrm{rim}$ area +3.7 rim volume +4.3 retinal nerve fibre layer -3.7 cup shape -3.1 cup volume -0.9 cup area. This score, including six HRT variables, was used to evaluate sensitivity and specificity with receiver operator characteristics curve. The overall score (D) including all six

Table 3 Sensitivity and specificity of morphometric optic disc variables, measured by confocal laser scanning tomography, in the differentiation of normal eyes, eyes with preperimetric glaucoma, and eyes with perimetric glaucoma. $p$ Values, calculated for the significance of differences to the normal group (ranked Mann-Whitney $U$ test). All variables of the perimetric glaucoma group were significantly reduced $(p<0.001)$

\begin{tabular}{|c|c|c|c|c|}
\hline HRT variable & Region & $\begin{array}{l}\text { Mann-Whitney test ( } p \\
\text { value) preperimetric } O A G\end{array}$ & $\begin{array}{l}\text { Sensitivity (\%) (specificity } \\
95 \% \text { ) preperimetric OAG }\end{array}$ & $\begin{array}{l}\text { Sensitivity (\%) } \\
\text { (specificity 95\%) } \\
\text { perimetric OAG }\end{array}$ \\
\hline \multirow[t]{3}{*}{ Rim area } & all & 0.02 & 11.8 & 65.6 \\
\hline & superior & 0.001 & 24.8 & 72.1 \\
\hline & inferior & ns & 8.8 & 57.4 \\
\hline \multirow[t]{3}{*}{ Rim volume } & all & 0.0002 & 19.6 & 67.2 \\
\hline & superior & 0.0001 & 25.5 & 73.8 \\
\hline & inferior & 0.002 & 11.8 & 60.7 \\
\hline \multirow[t]{3}{*}{ RNF thickness } & all & 0.004 & 19.6 & 54.1 \\
\hline & superior & 0.007 & 18.6 & 50.8 \\
\hline & inferior & 0.04 & 26.5 & 60.7 \\
\hline \multirow[t]{3}{*}{ Cup area } & all & 0.04 & 13.7 & 57.4 \\
\hline & superior & 0.02 & 13.7 & 60.7 \\
\hline & inferior & ns & 5.9 & 36.1 \\
\hline \multirow[t]{3}{*}{ Cup volume } & all & ns & 15.7 & 49.2 \\
\hline & superior & 0.02 & 23.5 & 60.7 \\
\hline & inferior & ns & 13.7 & 49.2 \\
\hline \multirow[t]{3}{*}{ Cup shape measure } & all & ns & 9.8 & 42.6 \\
\hline & superior & 0.004 & 7.8 & 36.1 \\
\hline & inferior & & 2.9 & 26.2 \\
\hline Multivariate analysis & & $<0.0001$ & 42.2 & 83.6 \\
\hline
\end{tabular}


measurements reached a sensitivity of $83.6 \%$ at a specificity of $95 \%$. It means that the combination of the six optic disc variables had a higher diagnostic power than each of the variables taken alone. For the differentiation of the normal eyes and eyes with preperimetric glaucoma, the same multivariate approach increased the sensitivity value to $42.2 \%$ at $95 \%$ specificity (Table 3 ).

\section{Discussion}

Traditionally glaucoma has been defined by the triad of increased IOP, optic disc changes, and visual field defects. Histological studies have shown however that there can be a significant loss of ganglion cells before evidence of functional loss on conventional achromatic visual field testing. ${ }^{14}{ }^{15}$ For this reason attention has been focused on alternative, more sensitive ways of detecting early ganglion cell damage than it is possible with white on white perimetry. In several studies on eyes with elevated intraocular pressure and normal visual fields, abnormal results in various psychophysical and electrophysical examinations were reported. ${ }^{16-21}$ As with the newer psychophysical and electrophysiological techniques, it has been shown that abnormalities in the appearance of the optic disc may precede visual field defects. ${ }^{4-9} 2223$ These abnormalities include an unusually small area of the neuroretinal rim, an abnormal shape of the rim, high cup to disc ratios, an usually large parapapillary chorioretinal atrophy, a decreased visibility of the retinal nerve fibre layer including localised defects, and the presence of splinter-shaped haemorrhages at the optic disc border. The variables with the highest predictive value to differentiate between normal eyes and eyes with increased IOP and normal visual fields were shape of the neuroretinal rim and visibility of the retinal nerve fibre layer. ${ }^{9}$ Presence of localised defects of the retinal nerve fibre layer and splinter-shaped disc haemorrhages had high specificities; however, their sensitivity was relatively low.

Most of these variables are qualitative or semiquantitative. They can be evaluated by the ophthalmoscopy without the necessity of measuring them. In an automated analysis of the optic nerve head, however, quantitative variables are determined such as area and volume of the neuroretinal rim and depth of the optic disc cup. With confocal laser scanning tomography as a technique for computerised analysis of the optic nerve head becoming more popular, ${ }^{11}{ }^{12} 24-28$ the purpose of the present study was to evaluate sensitivity and specificity of quantitative optic disc variables in the differentiation between normal eyes and eyes with increased IOP and normal visual fields.

In the normal group, we found a pronounced interindividual variability for all optic disc variables measured (Table 2) (Figs 1-3). Similar results have been obtained when the optic disc variables were measured by planimetry of stereo optic disc photographs. ${ }^{1329}$ The interindividual variability of the HRT data may be caused by the heterogeneity of a
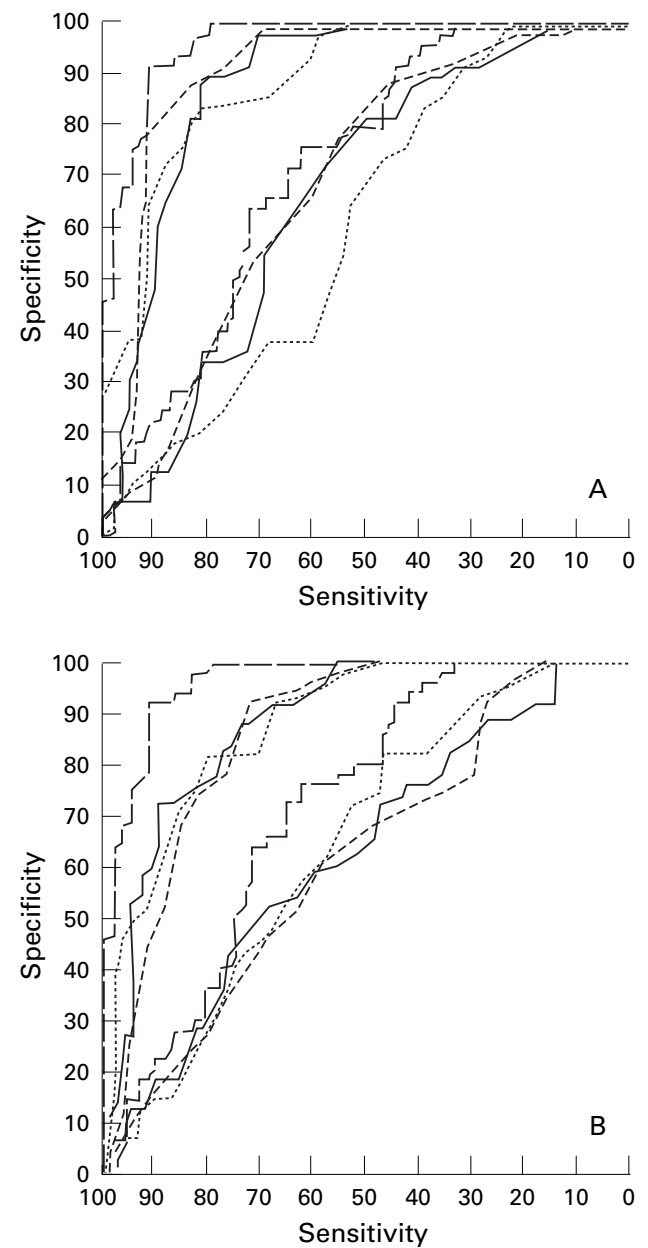

Figure 4 ROC (receiver operated characteristic) curves generated for multivariate analysis (broken line) and optic disc variables measured by confocal laser scanning tomography in the preperimetric and perimetric glaucoma group. (A) Variables of the rim: rim area superior (more broken line), rim volume superior (solid line), RNF inferior (dotted line). (B) Variables of the cup: cup volume superior (broken line), cup area superior (solid line), cup shape measure (dotted line).

biological group, the influence of methodological factors such as varying conditions in taking the HRT images, or the observer associated variability in defining the disc margin, or by other factors. The marked interindividiual variability being typical also for many other quantitative biological variables such as body height and weight may be the reason why the normal group and the preperimetric glaucoma group showed a pronounced overlap in the quantitative optic disc variables (Figs 1-3). Correspondingly, sensitivity and specificity of these variables were relatively low to differentiate between the normal eyes and the eyes with preperimetric glaucoma (Table 3) (Figs 1-3, Fig 4). To cite an example, the optic disc variable rim volume above reference superior detected glaucomatous optic nerve damage in $26 \%$ of the preperimetric eyes at a given specificity of $95 \%$, although all of these eyes showed glaucomatous optic nerve damage as described by qualitative optic disc variables. It shows that, by taking only one quantitative optic disc variable, it is rather difficult to detect early glaucomatous optic nerve damage. 
It suggests that the combination of several quantitative disc variables in the form of a discriminant analysis should increase the likelihood of detecting glaucomatous abnormalities in optic nerve heads which, by ophthalmoscopic evaluation, show qualitative abnormalities such as presence of localised retinal nerve fibre defects. In the present study discriminant analysis increased sensitivity in the preperimetric group from $27 \%$ for the best single variable (RNF thickness inferior) up to $42.2 \%$. In the group of perimetric glaucomatous optic discs sensitivity at $95 \%$ specificity increased from $73.8 \%$ for the single variable rim volume above reference superior to $83.6 \%$ by multivariate analysis.

The variables area and volume of the optic rim and cup and cup shape measure showed higher sensitivities in the superior than in the inferior sectors both in the preperimetric and the perimetric group at $95 \%$ specificity. These findings seem to stand in contrast with earlier reports showing pronounced rim loss in the inferior sector of the optic disc and perimetric field loss in the superior half of the visual filed in early glaucoma. ${ }^{22}{ }^{30-32}$ As pointed out earlier, the retinal vessels are included into the measurements of cup and disc by the HRT. ${ }^{27}$ One could speculate that the proportion of the vessels contributing to rim and cup volume is smaller in the superior sector, as the disc is scanned nearly perpendicularly and the values for the HRT variables are more valid.

The results of the present study confirm previous investigations in which confocal scanning laser tomography was used to measure quantitative optic disc variables in eyes with early glaucoma. ${ }^{26}{ }^{28}$ Defining early glaucomatous visual field loss as mean perimetric defects of less than $5 \mathrm{~dB}$, Mikelberg and co-workers ${ }^{26}$ reported highest sensitivity at $89 \%$ and $78 \%$ specificity using the papillomacular bundle as a reference plane and cup shape measure being one of the most informative variables. Ushida et al ${ }^{28}$ found $83 \%$ sensitivity at $86 \%$ specificity with the variable cup shape measure. In the present study, sensitivity and specificity values were lower than in the two other studies cited. The reason may be that glaucomatous optic nerve damage was less marked in the preperimetric group of the present study than in the groups of the investigations previously cited in which the glaucomatous eyes had manifest perimetric defects. As in the present study, Mikelberg and others ${ }^{26}{ }^{28}$ also found that a combination of optic disc variables in the form of a multivariate approach improves the discrimination between normal and glaucomatous eyes.

The present cross sectional study did not evaluate the ability of the HRT in detecting change in follow up examination, which has already clearly been demonstrated by Hatch and co-workers. ${ }^{34}$ Future studies may reveal whether quantitative data of the optic disc and retinal nerve fibre layer as measured by other techniques such as planimetry of the optic disc photographs, ${ }^{13}{ }^{29}$ polarimetry, ${ }^{35}$ or optical coherence tomography ${ }^{36}$ have a higher diagnostic power than optic disc variables determined by confocal scanning laser tomography, or whether quantitative optic disc variables are generally more useful for follow up examination of patients ${ }^{34}$ than for the detection of glaucomatous optic nerve damage in a cross sectional evaluation.

Supported by Deutsche Forschungsgemeinschaft (SFB 539). Proprietary interest: none.

1 Airaksinen PJ, Tuulonen A, Werner EB. Clinical evaluation of the optic disc and retinal nerve fiber layer. In: Ritch R, Shields MB, Krupin T, eds. The glaucomas. St Louis: Mosby, 1996:617-58.

2 Shields MB. Textbook of glaucoma. Baltimore: Williams and Wilkins, 1998:72-107.

3 Jonas JB, Nguyen NX, Naumann GOH. Non-quantitative morphologic criteria in normal and glaucomatous optic discs. Acta Ophthalmol 1989;67:361-6.

4 Sommer A, Pollack I, Maumenee AE. Optic disc parameters and onset of glaucomatous visual field loss. I. Methods and progressive changes in disc morphology. Arch Ophthalmol 1979;97:1444-8.

5 Pederson JE, Anderson DR. The mode of progressive disc cupping in ocular hypertension and glaucoma. Arch 980;98:490-5

6 Balazsi AG, Drance SM, Schulzer M, et al. Neuroretinal rim area in suspected glaucoma and early open-angle glaucoma. Correlations with parameters of visual function. Arch Ophthalmol 1984;102:1011-4.

7 Caprioli J, Miller JM, Sears M. Quantitative evaluation of the optic nerve head in patients with unilateral visual field loss from primary open-angle glaucoma. Ophthalmology 1987;94:1484-7.

8 Tuulonen A, Lehtola J, Airaksinen PJ. Nerve fiber layer defects with normal visual fields. Ophthalmology 1993;100: $587-98$.

9 Jonas JB, Königsreuther KA. Optic disk appearance in ocular hypertensive eyes. Am f Ophthalmol 1994;117:732-40.

10 Jonas JB, Dichtl A. Optic disc morphology in myopic primary open-angle glaucoma. Graefes Arch Clin Exp Ophthalmol 1997;235:627-33.

11 Kruse FE, Burk ROW, Völcker HE, et al. Reproducibility of topographic measurements of the optic nerve head with laser tomographic scanning. Ophthalmology 1989;96:13204.

12 Burk ROW, Rohrschneider K, Takamoto T, et al. Laser scanning tomography and stereogrammetry in three dimensional optic disc analysis. Graefes Arch Clin Exp Ophthalmol 1993;231:193-8.

13 Jonas JB, Fernandez MC, Naumann GOH. Glaucomatous parapapillary atrophy: occurrence and correlations. Arch Ophthalmol 1992;110:214-22.

14 Quigley HA, Addicks EM, Green WR, et al. Optic nerve damage in human glaucoma. III. Quantitative correlation of nerve fiber loss and visual field defect in glaucoma, ischemic optic neuropathy, papilledema and toxic neuropathy. Arch Ophthalmol 1989;107:453-64.

15 Quigley HA, Dunkelberger GR, Green WR. Retinal ganglion cell atrophy correlated with automated perimetry in human eyes with glaucoma. Am f Ophthalmol 1989;107: in human 64 .

16 Kohn AN, Moss AP, Podos SM. Relative afferent pupillary defects in glaucoma without characteristic field loss. Arch defects in glaucoma without

17 Tyler CW. Specific deficits of flicker sensitivity in glaucoma and ocular hypertension. Invest Ophthalmol Vis Sci 1981;20:204-12

18 Glovinsky Y, Quigley HA, Drum B, et al. A whole field scotopic retinal sensitivity test for the detection of early glaucomatous damage. Arch Ophthalmol 1992;110:486-90.

19 Sample PM, Martinez GA, Weinreb RN. Short wavelength automated perimetry without lens density testing. Am 7 Ophthalmol 1994;118:632-41.

20 Horn F, Jonas JB, Jünemann A, et al. The full field flicker test in early diagnosis of chronic open-angle glaucoma. Am f Ophthalmol 1997;123:313-9.

21 Teesalu P, Vihanninkijo K, Airaksinen PJ, et al. Correlation of blue-on-yellow visual fields with scanning confocal laser optic disc meas

22 Sommer A, Katz J, Quigley HA, et al. Clinically detectable nerve fiber atrophy precedes the onset of glaucomatous field loss. Arch Ophthalmol 1991;109:77-83.

23 Zeyen TG, Caprioli J. Progression of disc and field damage in early glaucoma. Arch Ophthalmol 1993;111:62-5.

24 Dreher AW, Tso PC, Weinreb RN. Reproducibility of topographic measurements of the normal and glaucomatous nerve head with the laser tomographic scanner. $\mathrm{Am} \mathcal{F} \mathrm{Oph}$ thalmol 1991;111:221-9.

25 Rohrschneider K, Burk ROW, Völcker HE. Reproducibility of topometric data acquisition in normal and glaucomatous optic nerve heads with the laser tomographic scanner. optic nerve heads with the laser tomographic scan
Graefes Arch Clin Exp Ophthalmol 1993;231:457-64.

26 Mikelberg FS, Parfitt CM, Swindale NV, et al. Ability of the Heidelberg retina tomograph to detect early glaucomatous field loss. F Glaucoma 1995;4:242-7. 
27 Dichtl A, Jonas JB, Mardin CY. Comparison between tomographic scanning evaluation and photographic measurement of the neuroretinal rim Am 7 Ophthalmol 1996;121: 494-501.

28 Uchida H, Brigatti L, Caprioli J. Detection of structural damage from glaucoma with confocal laser image analysis. Invest Ophthalmol 1996:37:2393-401.

29 Jonas JB, Gusek GC, Naumann GOH. Optic disc morphometry in chronic open-angle glaucoma. I. Morphometric intrapapillary characteristics. Graefes Arch Clin Exp Ophthalmol 1988;226:522-30.

30 Tuulonen A. The morphological pattern of early glaucomatous damage. Curr Opin Ophthalmol 1993;4:29-34

31 Gramer E, Gerlach R, Krieglstein GK, et al. Zur Topographie früher glaukomatöser Gesichtsfeldausfälle bei der Computerperimetrie. Klin Monatsbl Augenheilkd 1982; 180:490-5.
32 Airaksinen PJ, Drance SM, Douglas GR, et al. Visual field and retinal nerve fiber layer comparisons in glaucoma. Arch 1985:103:205-7.

33 Bathija R, Zangwill L, Berry CC, et al. Detection of early glaucomatous structural damage with confocal scanning laser tomography. F Glaucoma 1998;7:121-7.

34 Hatch WV, Flanagan JG, Etchells EE, et al. Laser scanning tomography of the optic nerve head in ocular hypertension and glaucoma. Br f Ophthalmol 1997;81:871-6.

35 Tjon-Fo-Sang MJ, de Vries J, Lemji HG. Measurement by nerve fiber analyzer of retinal nerve fiber layer thickness in normal subjects and patients with ocular hypertension. Am 7 Ophthalmol 1996;122:220-7.

36 Schumann JS, Hee MR, Puliafito CA, et al. Quantification of nerve fiber layer thickness in normal and glaucomatous eyes using optical coherence tomography. Arch Ophthalmol 1995;113:586-96. 